\title{
Numerical investigation using an exact solution of the effects of non-solenoidality of the viscous terms on the incompressible flow
}

\author{
Hiroki SUZUKI*, Shingo HATTORI* and Shinsuke MOCHIZUKI* \\ * Graduate School of Sciences and Technology for Innovation, Yamaguchi University \\ 2-16-1 Tokiwa-dai, Ube-shi, Yamaguchi 755-8611, Japan \\ E-mail: h.suzuki@yamaguchi-u.ac.jp
}

Received: 21 April 2016; Revised: 30 November 2016; Accepted: 12 January 2017

\begin{abstract}
We examine effects of the divergence of the viscous terms on the numerical results of an incompressible flow by using an exact solution of the governing equation. When the Poisson equation is solved using fractional steps, the divergence in the velocity field may have nonzero magnitude. It should be noted that the divergence of the viscous terms can be larger than that of the velocity field. We use an exact solution, which is commonly used for benchmarking, to examine the effects of the divergence of the viscous terms. The divergence of the viscous terms affects the equilibrium relations found in the exact solution. In the present numerical results, the divergence of the viscous terms reduces the rate of decay of the kinetic energy and the pressure, and it also causes some harmonic waves in the flow field. We examine these results quantitatively by using approximations to the numerical results. Skewness and kurtosis factors of the physical quantities are also affected by the divergence of the viscous terms. These results show that the divergence of the viscous terms significantly affects the flow field.
\end{abstract}

Key words : Numerical simulation, Incompressible flow, Viscous terms, Exact solution, Poisson equation

\section{Introduction}

A large-eddy simulation (LES) is a common approach to finding a numerical solution to an incompressible flow. LES is a useful method for solving fluid problems in engineering applications, although the computational cost of direct numerical simulation (DNS) may be prohibitively large. Recently, Quasi-DNS has been proposed (Komen et al., 2014; Addad et al., 2015) as a method that is intermediate between DNS and LES; this kind of LES uses finite-difference approximations. Fractional step methods (e.g., Kim and Moin, 1985) are commonly used in simulations for incompressible flows, and they require solving the Poisson equation. Although the direct methods for solving the Poisson equation are efficient (these are often based on the fast Fourier transform), these methods can be used only for some types of flow. In most engineering flow problems, an iterative method is used to solve the Poisson equation (e.g., Kato et al., 2000).

With both DNS and LES, various discretization schemes have been considered for the viscous and diffusion terms and the spatial resolution; this is necessary because the viscous and diffusion terms dominate the dissipation of a turbulent flow, which is large when the wavenumber is high. Miyauchi et al. (1994) showed the applicability of higher-order discretization schemes for viscous terms in a two-dimensional flow. Lamballais et al. (2011) showed higher-order numerical dissipation by applying compact schemes to discretized viscous terms. Gotoh et al. (2012) reduced the computational cost of direct simulation of the passive scalar in isotropic turbulence with a high Schmidt number; they did this by creating a discretization scheme for the diffusion terms in the convection-diffusion equation. Suzuki et al. (2013a) improved the accuracy of the higher-order statistics and spectra in a turbulent channel flow by applying compact schemes to the discretization of the viscous terms. In some previous DNSs (Suzuki et al., 2010; Suzuki et al., 2013b; Zhou et al., 2014a, 2014b), a finite-difference approximation for discretizing viscous terms resulted in greater accuracy than did an approximation for discretizing convection terms. In an LES, it is important to ensure that the spatial resolution is sufficient, because the magnitude of the discretization error is comparable to that of subgrid scale (SGS) stress. 
We thus assume that the effect of the divergence in the viscous term should be taken into account when simulating an incompressible flow. Iteration methods, such as the successive overrelaxation (SOR), conjugate gradient (CG) and biconjugate gradient stabilized (BiCGstab) methods, are often used to solve the Poisson equation in an unsteady numerical simulation of an incompressible flow. These methods are common components of so-called computational fluid dynamics (CFD) solvers. In the numerical simulation of an incompressible flow, it is important that the grid spacing and time increment be sufficiently small, and the iteration number be sufficiently large. The value of these computational parameters is directly related to the cost of the numerical simulation. In most simulations of incompressible flows, the cost of solving the Poisson equation accounts for the bulk of the cost of the entire simulation. In this approach, reducing the number of iterations increases the uncertainty in the velocity divergence. Reducing the number of iterations could notably decrease the cost of the numerical simulations. We believe it is important to investigate the effects of a limited number of iterations on the observed flow field. Attempts to improve the efficiency of solving the Poisson equation have been made. Tamura et al. (1997) proposed a residual-cutting method for solving the Poisson equation, and they examined the validity of the method. Morinishi et al. (2011) proposed a preprocessing scheme for solving the Poisson equation. In numerical measurements of incompressible flows, the divergence of the viscous terms should be zero, since the velocity divergence has zero magnitude. Therefore, when the velocity divergence deviates from zero during the application of an iteration method, the magnitude of the viscous terms will also be nonzero. The formulation of the viscous term is based on the second-order differential term of the velocity field and includes the Reynolds number. The magnitude of the second-order differential term will increase as the grid spacing decreases. Therefore, when both the Reynolds number and the grid spacing are small, the magnitude of the divergence in the viscous term can be larger than that of the velocity divergence. Although the effect of the divergence in the viscous term on the characteristics of an incompressible flow could be large, previous numerical studies that have considered the discretization of the viscous term have focused on the accuracy of the discretization scheme and the effective wave number of the scheme.

The purpose of this study is to examine the effect of non-solenoidality in the viscous term on the incompressible flow. We use an exact solution to solve this problem. We present a numerical simulation that uses an iteration method that solves the Poisson equation in fractional steps. In our simulated flow, the divergence in the viscous term is larger than the velocity divergence. We examine the effect of the divergence in the viscous term by using a flow that is obtained as an exact solution. Exact solutions (e.g., Hui, 1987; Wang, 1990) are often used for benchmarking numerical simulations (Wang, 1991). The structures of a flow described by an exact solution are known exactly and can be formulated. Therefore, using an exact solution to solve the present problem will clarify the effect due to the divergence. Dissipation of kinetic energy in turbulent flows is greater when the wavenumber is larger, and it arises due to the viscous term. When the divergence in the viscous term is significant, it will increase the dissipation of kinetic energy; thus, it affects the rate of change in kinetic energy. Therefore, we focus on the effect of the divergence on the decrease in kinetic energy. The nonzero divergence of the viscous term is due to the velocity divergence; therefore, the divergence of the velocity and that of the viscous terms are nonzero in the present numerical simulations. It should be noted that the divergence of the viscous terms increases as the grid spacing decreases. Therefore, with fine spatial resolution, the divergence of the viscous term could be significantly larger than that of the velocity field. Note also that, in the present simulation, the uncertainty in the velocity divergence does not affect the kinetic energy when the flow field is inviscid, because the skew-symmetric form is used to analyze the convective terms. Based on these factors, we consider that, in the present study, the error in the observed flow field is primarily due to the divergence of the viscous terms.

\section{Methods}

\subsection{Numerical simulation}

We will now describe the flow that will be simulated and the methods that will be used. The governing equations are the continuity equation and the Navier-Stokes equations for incompressible flows; we will use the two-dimensional nondimensional form. The characteristic velocity and length scales are $\sqrt{K_{o}}$ and $\eta$, respectively, where $K_{o}$ is the dimensional global kinetic energy per unit area in the initial state, and $2 \pi \eta$ is the wavelength of the exact solution (see the next section). The nondimensional coordinate system consists of $t, x$ and $y$, where $t$ is the nondimensional time, and $x$ and $y$ are nondimensional spatial coordinates. The nondimensional physical quantities in the governing equations are $u$ and $v$, which are the velocities in the $x$ and $y$ directions, respectively, and the pressure $p$. A low-storage fourth-order Runge-Kutta scheme (RK4) is used for the temporal integration of the governing equation. Various other methods are also used in the verification of the present simulation. Because we calculate the uncertainty in the kinetic energy conservation due to the temporal integration scheme, we also use the first-order Euler scheme (E1) and the third-order low-storage Runge-Kutta 
(a)

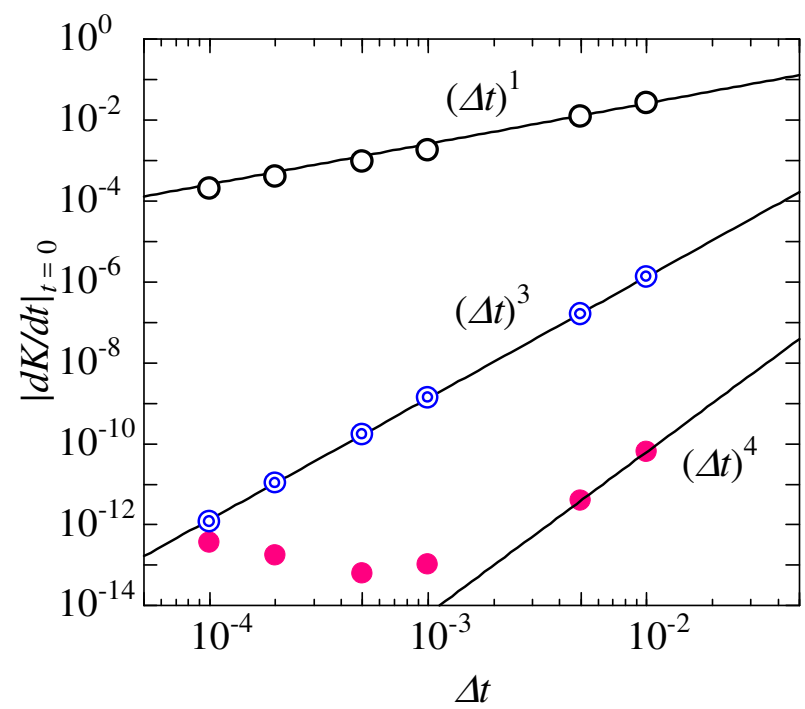

(b)

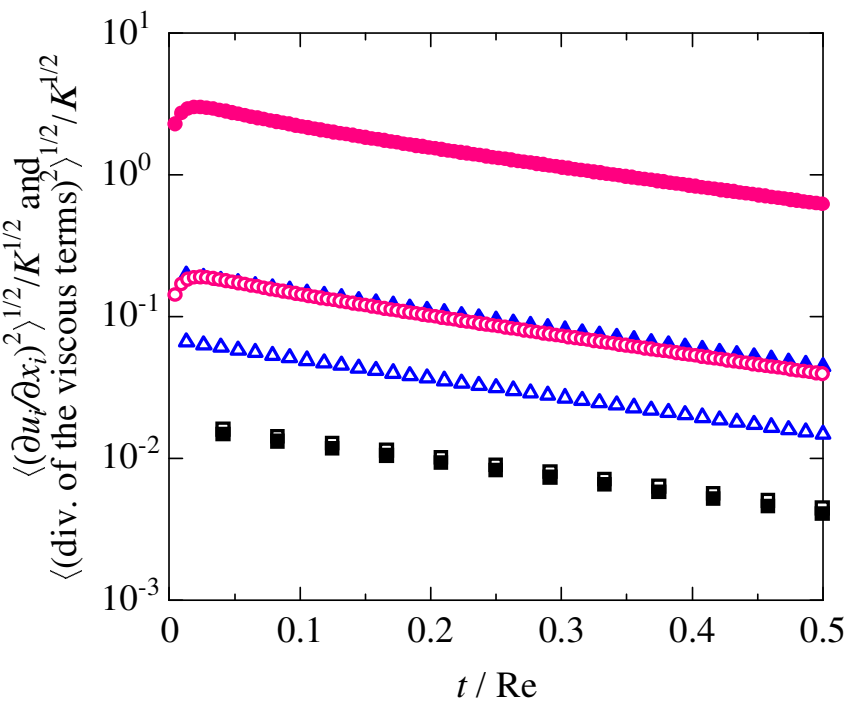

Fig. 1 Results of the code verification and the divergence of the velocity and viscous terms. (a) Results of the code verification: red filled circles, blue double circles and open black circles show the errors in the kinetic energy caused by RK4, RK3 and E1, respectively. These results agree with the analytical results, and thus they verify our proposed method. The kinetic energy has the smallest error when RK4 was used. (b) rms of the divergence of the velocity and viscous terms, normalized by the square root of the kinetic energy; $\epsilon_{p}=1$. Here red, blue and black symbols show the results for $N=128,48$ and 16, respectively. Open symbols show the divergence of the velocity, and filled symbols show the divergence of the viscous terms. When $N=16$, the divergence of the viscous terms is comparable to that of the velocity. The divergence of the viscous terms can be larger than that of the velocity, as can be seen when $N=128$. The difference in the divergences increases as the spatial resolution becomes finer.

scheme (RK3). The algorithm we use to simultaneously solve the governing equations is the fractional step method. The Poisson equation was solved by using SOR with fractional steps. The number of iterations was based on a measure of the convergence condition $\epsilon_{p}: \epsilon_{p}=\left\langle\left\|p^{m+1}-p^{m}\right\|\right\rangle /\left\langle\left\|p^{m+1}\right\|\right\rangle$, where $m$ is the number of iterations.

The choice of discretization scheme for the convective terms is important, because we wish to study the effect of the divergence in the viscous terms on the exact solution: it is important to choose a discretization scheme that has a negligible conservation error for the kinetic energy. Therefore, we used the skew-symmetric form proposed by Morinishi et al. (1998) to discretize the convective terms. In the flow solvers that are in common use, a second-order scheme would be used for the discretization of the convective terms (e.g., Kato et al., 2000); therefore, we use a second-order discretization scheme in the present simulation. We also use a second-order discretization of the viscous terms, because the accuracy of the spatial discretization is often the same for both the convection and the viscous terms (e.g., Kato et al., 2000).

The area of the square computational domain is $L^{2}=(4 \pi)^{2}$. The number of grid points is set to $N^{2}=16^{2}, 24^{2}, 32^{2}$, $48^{2}, 64^{2}, 96^{2}$, or $128^{2}$. Instead of choosing the temporal increment $\Delta t$, we set the value of the Courant-Friedrich-Lewy condition (CFL), which is defined by taking the velocity as unity: CFL $=\pi^{2} / 32, \pi^{2} / 64$, or $\pi^{2} / 96$. The Reynolds number was set to $2 \pi$, and therefore, the maximum value of the diffusion number is $1 / 2$. For our purpose, the magnitude of the divergence in the viscous terms should be varied, so we used the range $\epsilon_{p}=1-0.001$. In the initial state, the flow field is the exact solution; the flow field is simulated up to $t=\pi$.

We verify our proposed numerical algorithm and examine the uncertainty in the kinetic energy. We do this by simulating a homogeneous isotropic inviscid flow (Morinishi et al., 1998). The initial velocity is a random field that satisfies the continuity equation. Figure 1(a) shows the rate of change of the global kinetic energy per unit area at the initial state $|d K / d t|_{t=0}$. This rate is formulated analytically as $|d K / d t|_{t=0}=\alpha \Delta t^{n_{i}}$, where $\alpha$ is a coefficient, $\Delta t$ is the time increment, and $n_{i}$ is the accuracy of the time integration. The obtained uncertainty agrees with the corresponding analytical formula, and this verifies our proposed algorithm. The uncertainty of $|d K / d t|_{t=0}$ is negligibly small when using RK4. 
(a)

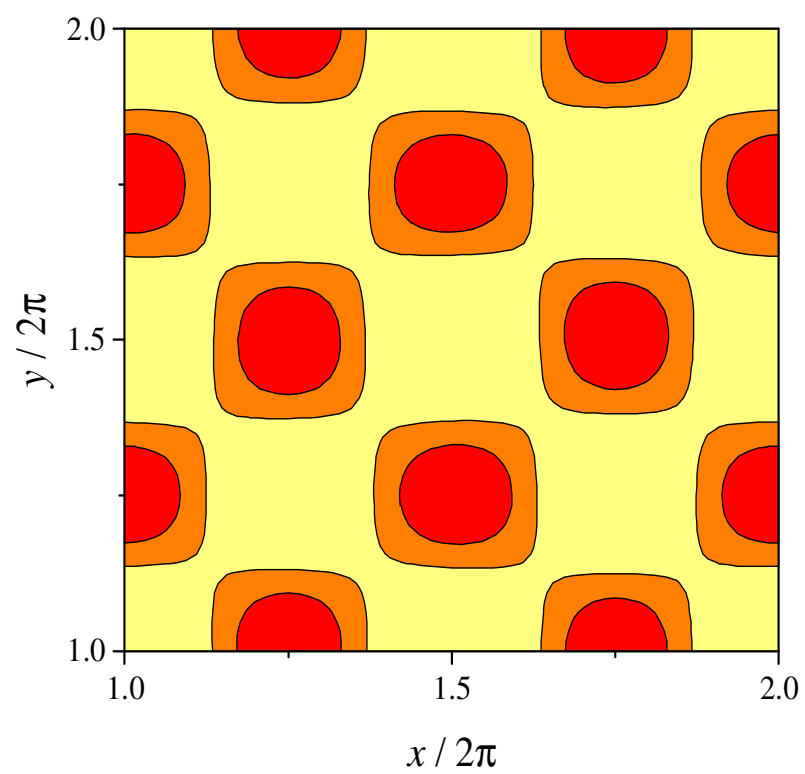

(b)

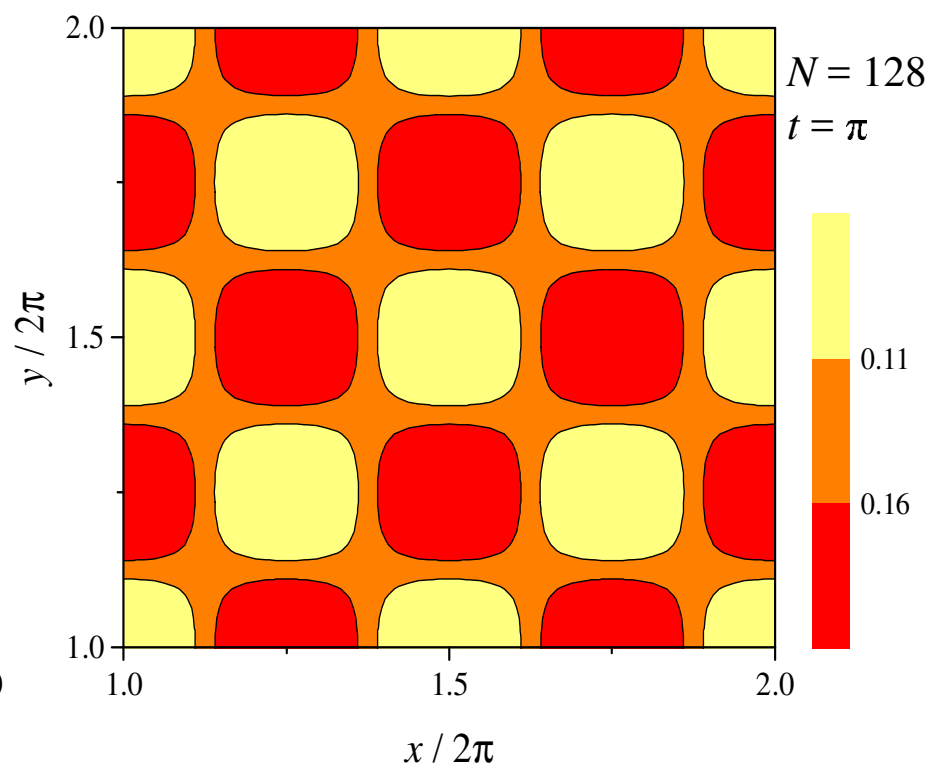

Fig. 2 Results of flow visualization. The kinetic energy of the numerical result and that of the exact solution are shown in (a) and (b), respectively; here, $N=128$ and $t=\pi$. The meaning of the coloring is shown at the right. The yellow region in the numerical result is larger than that in the exact solution, and this suggests a decrease in the global kinetic energy, due to divergence. We can see that the flow pattern of the numerical result is distorted, and this suggests that there is an additional flow field with a higher wave number.

\subsection{Exact solution}

We use the exact solution to evaluate our approach, as is often done for benchmarking numerical simulations. The two-dimensional Taylor-Green vortex flow has commonly been used as an exact solution of incompressible flows (e.g., Rogler and Reshotko, 1976). Kim and Moin (1985) applied this exact solution to the validation of their scheme, and San and Staples (2013) used this flow as the first problem in their examination. We applied this exact solution to the present problem, which is formulated as follows:

$$
\begin{aligned}
& u(x, y, t)=-2 \cos (x) \sin (y) \exp (-2 t / \mathrm{Re}), \\
& v(x, y, t)=2 \sin (x) \cos (y) \exp (-2 t / \mathrm{Re}), \text { and } \\
& p(x, y, t)=(1 / 2)[\cos (2 x)+\cos (2 y)] \exp (-4 t / \mathrm{Re})
\end{aligned}
$$

We did not use the three-dimensional flow of Ethier and Steinman (1994), because even though it has the same equilibrium characteristics as those of the Taylor-Green vortex flow, the three-dimensional flow is not periodic, and it is difficult to determine the boundary conditions. Although a three-dimensional Taylor-Green vortex flow has been used to benchmark a similar numerical simulation (e.g., Brachet et al., 1983; Brachet, 1991), we do not use one here because we wish to simplify the effect of the divergence; unlike the two-dimensional case, this three-dimensional vortex flow has a global anisotropy. The advantage of using an exact solution is that the flow field is known exactly, and therefore, the global characteristics of the flow quantities are also known. For example, the global kinetic energy in the periodical square domain, $K \times L^{2}$, is derived as follows:

$$
K \times L^{2}=\frac{1}{2}\left(\left\langle u^{2}\right\rangle+\left\langle v^{2}\right\rangle\right) \times L^{2}=\exp (-4 t / \mathrm{Re}) \times L^{2} .
$$

Here \langle\rangle denotes the ensemble average covering the periodic domain.

In the flow described by an exact solution, the flow characteristics are entirely known. The Navier-Stokes equation contains unsteady terms, convective terms, pressure terms, and viscous terms. In the flow of a Taylor-Green vortex, the unsteady terms balance the viscous terms, and the convective terms balance the pressure terms; these two equilibrium relations are independent of each other. This independence was the focus of a previous study that derived the exact three-dimensional solution (Ethier and Steinman, 1994). The first equilibrium relation consists of the unsteady terms and the viscous terms, and it is equal to the diffusion equation, which governs the amplitude of the flow field. The second equilibrium relation governs the profile of the velocity and pressure fields. This independence should remain in any 

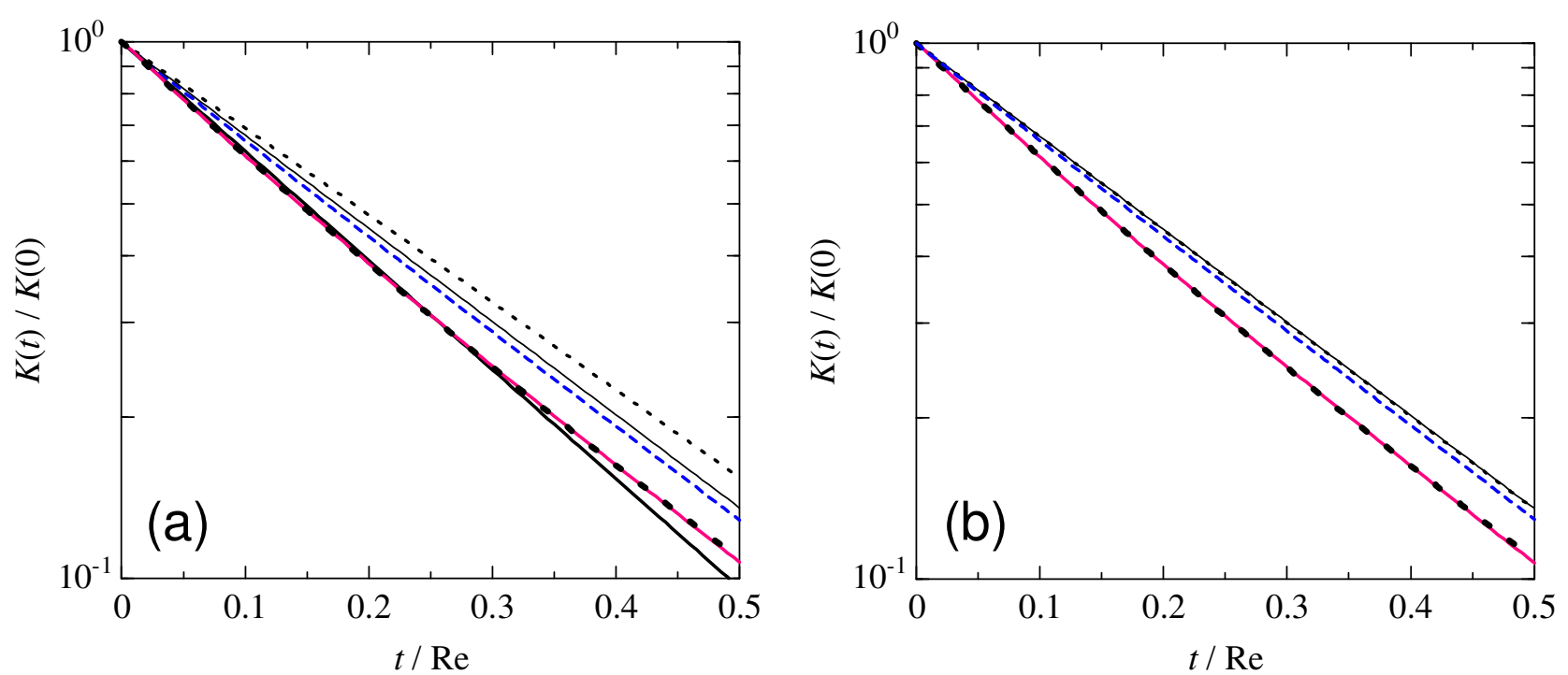

Fig. 3 Decay of global kinetic energy normalized by initial magnitude, $K(t) / K(0)$. The dependency of the grid number and $\epsilon_{p}$ are shown in (a) and (b), respectively. The thin lines show the exact solution shown as follows: (a) The red solid, blue dashed and black dotted lines show the results for $N=128,48$ and 16, respectively; $\epsilon_{p}=1$. (b) The red solid, blue dashed and black dotted lines show the results for $\epsilon_{p}=1,0.1$ and 0.01 , respectively; $N=128$. The line indicated by heavy dots shows Eq. (2) fit to the data. The thin solid line shows an exponential function fit to the profile.

equation that is derived from them. When the divergence of the viscous terms is not zero, the Poisson equation, which is derived from the equilibrium relation, cannot be derived. Therefore, the nonzero magnitude of the divergence in the viscous term will affect the independence of the two equilibrium relations.

We will show the results of the divergence in the viscous terms before presenting the numerical results. Figure 1(b) shows the temporal evolution of the divergence in the velocity and the viscous terms. Here, the magnitude of the divergence is normalized by the square of the global kinetic energy per unit time at $t, \sqrt{K}$. When the number of grid points is small (e.g., $N=16$ ), the divergence of the viscous terms is comparable to that of the velocity vector, but when the number of grid points is large (e.g., $N=128$ ), the divergence of the viscous terms can be greater than that of the velocity vector. Therefore, we assume that the divergence of the viscous term would increase as the spatial resolution improves. This large divergence will affect the flow field, which is the focus of our study. For example, the independence between the equilibrium relations found in the exact solution assumes that the divergence is negligible, and thus when the magnitude of the divergence of the viscous term is much greater than zero, this independence is compromised.

\section{Results}

\subsection{Decay characteristics}

Visualization of the flow fields shows that the divergence of the viscous terms affects the decay of the kinetic energy. Figure 2 shows the flow field of the kinetic energy for the numerical results and the exact solution for $t=\pi$. In the computational results, the divergence in the viscous terms is the largest when $N=128$ and $\epsilon_{p}=1$. In the flow field of the exact solution, regions of high and low kinetic energy alternate, and there is little variation in the kinetic energy in between these regions. In the numerical results, we can see that the region of low kinetic energy has expanded, and the area of the high kinetic energy has been reduced. These results suggest that the divergence of the viscous terms reduces the global kinetic energy. We also note that the flow patterns in the numerical results are slightly distorted, and this suggests that the flow field contains high-harmonic waves caused by the divergence of the viscous terms.

The divergence of the viscous terms reduces the kinetic energy of the flow. Figure 3(a) shows temporal profiles of the global kinetic energy per unit area, normalized by the initial value, $K(t) / K(0)$. The effect of spatial resolution on the kinetic energy can be seen clearly: when the spatial resolution is low $(N=16)$, the magnitude of the kinetic energy is larger than that of the exact solution. This is caused by underestimating the viscous terms when applying the finitedifference approximation, and it can be reduced by improving the spatial resolution. Note that when the spatial resolution is moderate $(N=48)$, the magnitude of the kinetic energy is only slightly smaller than that of the exact solution, which shows that the underestimation of the viscous terms can be reduced by improving the spatial resolution. The divergence of the viscous terms increases as the spatial resolution improves, which implies that a larger divergence affects the global 

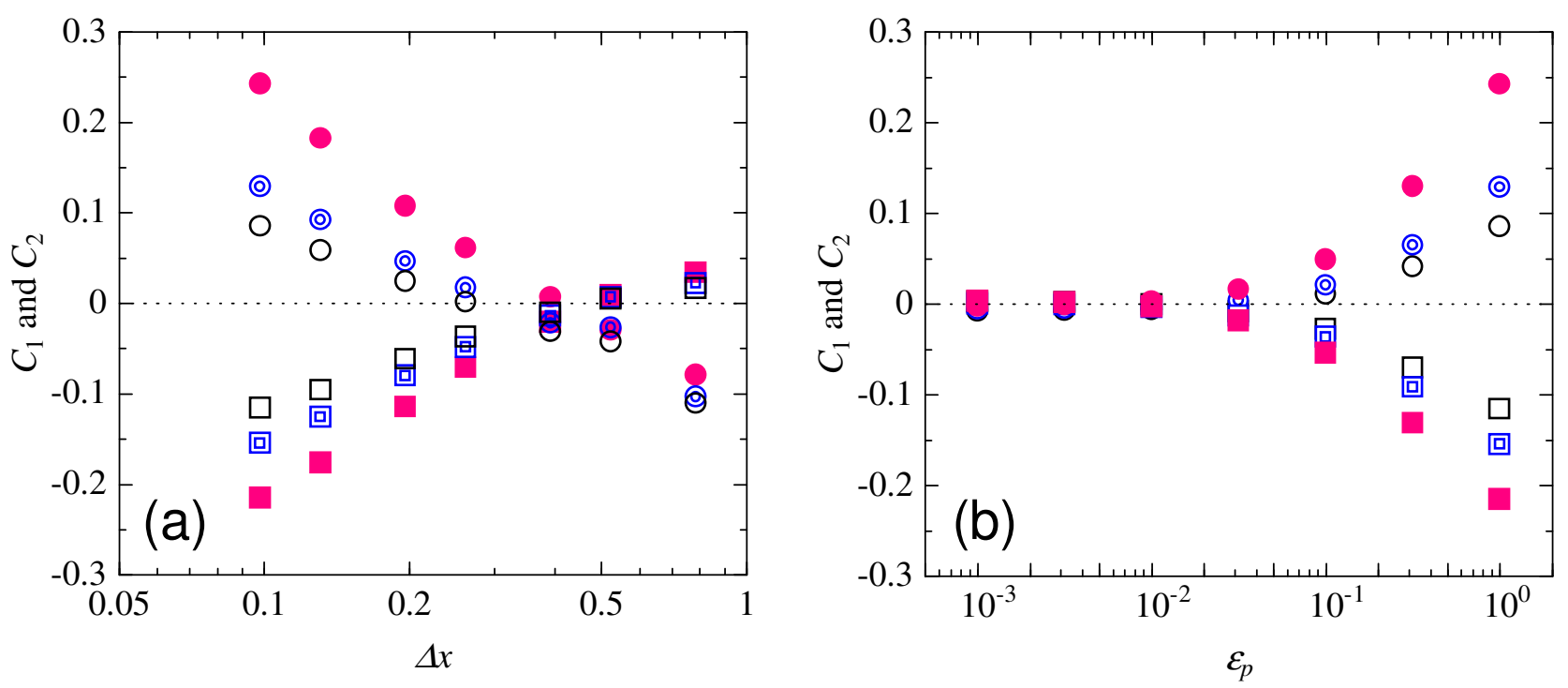

Fig. 4 Dependence of coefficients $C_{1}$ and $C_{2}$ on (a) the grid spacing $\Delta x$, and (b) the convergence condition $\epsilon_{p}$. In (a), $\epsilon_{p}=1$, and in (b), $N=128$. Here, $C_{1}$ and $C_{2}$ are indicated by circles and squares, respectively. Red filled, blue double and open black symbols show the results when the CFL condition is set to $\pi^{2} / 32$, $\pi^{2} / 64$ and $\pi^{2} / 96$, respectively; the deviation of the coefficients from zero is considered to be due to the uncertainty in the viscous terms. In (a), when $\Delta x$ is small, the absolute magnitude of the coefficients increases as the grid spacing decreases. The deviation of the coefficients decreases as $\epsilon_{p}$ decreases.

kinetic energy. When the spatial resolution is high $(N=128)$, we can see that the kinetic energy is further reduced, and we note that this decrease can become very large. This effect is reduced as convergence improves, as shown in Fig. 3(b). When $\epsilon_{p}=0.01$, numerical results for the kinetic energy are in close agreement with the exact solution.

We introduce an approximation formula for evaluating the way in which the global kinetic energy is affected by the divergence of the viscous terms. The global kinetic energy of the numerical results does not agree with that of the exact solution due to the divergence of the viscous terms. The exact solution of the global kinetic energy is given by Eq. (2), and we expected that the numerical results could be described by slightly modifying this exact solution. The solid black line in the Fig. 3(a) shows an exponential function that has been fitted to the numerical results. It can be seen that there is a large disagreement between this exponential function and the numerical results. Therefore, we introduce the following approximation:

$$
K(t) / K(0)=\exp \left[-\frac{4 t}{\operatorname{Re}}\left(1+C_{1}\right)\left(1+C_{2} \times \frac{t}{\operatorname{Re}}\right)\right],
$$

where $C_{1}$ and $C_{2}$ are coefficients that are equal to zero in the exact solution. These coefficients characterize the way in which the global kinetic energy is affected by the divergence of the viscous terms. Equation (3) expresses the numerical results with sufficient accuracy, as shown in Fig. 3.

The uncertainty characterized by the coefficients $C_{1}$ and $C_{2}$ is increased as the spatial resolution improves, as shown in Fig. 4. We show the dependence of these coefficients (a) on the grid spacing, $\Delta x$, and (b) on the convergence condition, $\epsilon_{p}$ in (b). When the spatial resolution is poor, $C_{1}$ and $C_{2}$ approach the exact value of zero as the spatial resolution improves. However, when the grid spacing is small, the deviation of the coefficient from the exact value increases as the grid spacing decreases. This increase in the deviation suggests that improving the spatial resolution increases the uncertainty in the observed flow field, and this is caused by the increased divergence of the viscous terms. When the divergence of the viscous terms is significant, $C_{1}$ is positive and $C_{2}$ is negative. Therefore, the divergence in the viscous terms increases the rate of decay of the global kinetic energy and also decreases the rate of change of this decay rate. The order of the value is the same between the coefficients $C_{1}$ and $C_{2}$. The deviation of the coefficients from the exact value decreases as CFL decreases, and it also decreases as convergence is approached, as shown in Fig. 4(b). When $\epsilon_{p}$ is on the order of 0.01, the deviation of the coefficients will be sufficiently small.

The pressure in the flow field is also affected by the divergence of the viscous terms. We examine the magnitude of the variation of the pressure field, normalized by the initial value $\left\langle p^{2}\right\rangle^{1 / 2} /\left.\left\langle p^{2}\right\rangle^{1 / 2}\right|_{t=0}$; this is shown in Fig. 5(a). Here $N=128$, and the temporal evolution of the pressure amplitude depends on the value of $\epsilon_{p}$. When $\epsilon_{p}$ is not small, the divergence of the viscous terms is significant because of the high spatial resolution, the pressure amplitude increases around the 

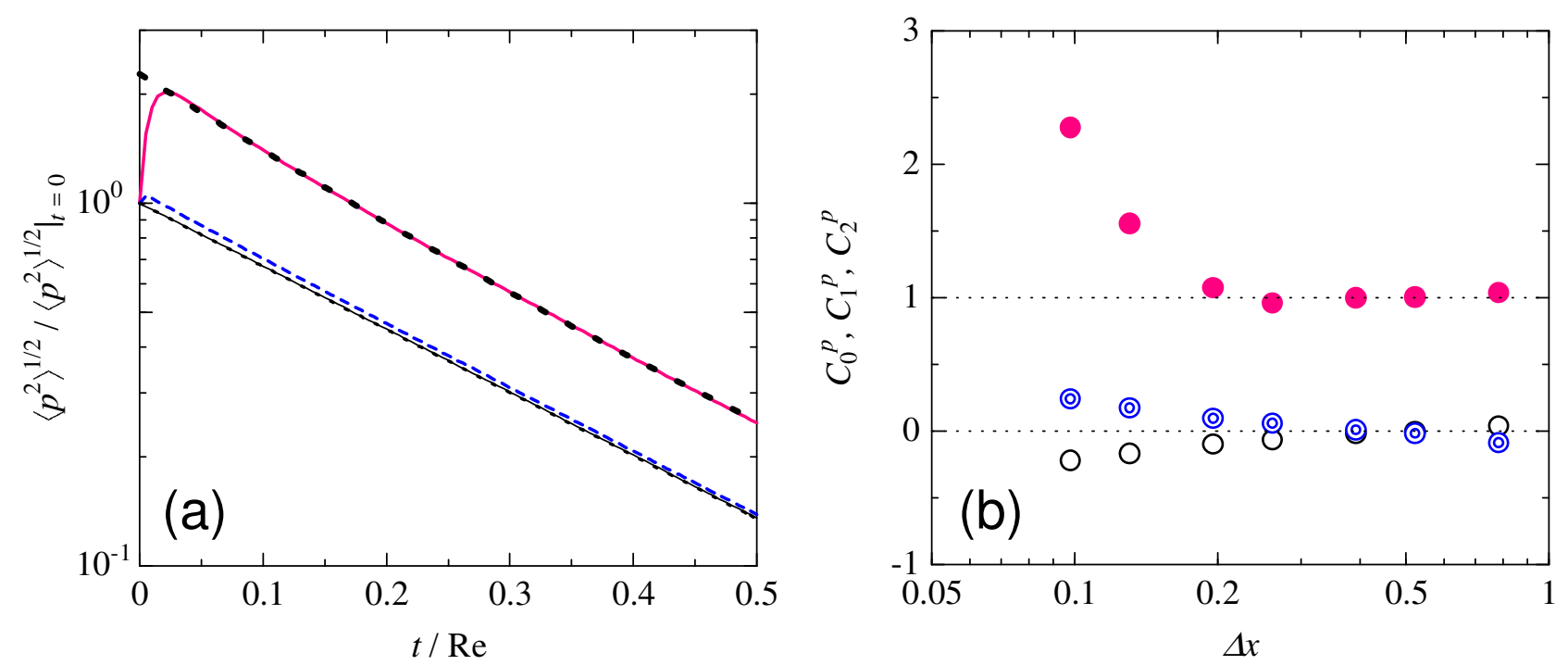

Fig. $5\left\langle p^{2}\right\rangle^{1 / 2} /\left.\left\langle p^{2}\right\rangle^{1 / 2}\right|_{t=0}$. Here, $N=128$ and $\epsilon_{p}=1$, and the magnitude of the divergence in the viscous terms is greatest. (a) Temporal profiles of this normalized pressure amplitude. Red solid, blue dashed and black dotted lines show the results for $\epsilon_{p}=1,0.1$ and 0.01 , respectively. The thin black line shows the exact profile. The divergence in the viscous terms causes an increase in $\left\langle p^{2}\right\rangle^{1 / 2} /\left.\left\langle p^{2}\right\rangle^{1 / 2}\right|_{t=0}$. The thick dotted line shows an approximation of Eq. (4), which is fitted to the profile; the approximation includes three coefficients, $C_{0}^{p}, C_{1}^{p}$ and $C_{2}^{p}$. (b) Dependency of these three coefficients on $\epsilon_{p}$. Red filled circles, blue double circles and open black circles show the results for $C_{0}^{p}, C_{1}^{p}$ and $C_{2}^{p}$, respectively. Of the coefficients, $C_{0}^{p}$ is the most sensitive to the divergence of the viscous terms. The value of each of the three coefficients approaches the exact value as $\epsilon_{p}$ decreases.

initial state, and this results in larger value than that of the exact evolution. This increase is reduced as convergence is approached. When $\epsilon_{p}$ is 0.01 , the numerical results agree well with those of the exact solution. We introduce the following approximation for the way in which the pressure amplitude is affected by the divergence of the viscous terms:

$$
\left\langle p^{2}\right\rangle^{1 / 2} /\left.\left\langle p^{2}\right\rangle^{1 / 2}\right|_{t=0}=C_{0}^{p} \exp \left[-\frac{4 t}{\operatorname{Re}}\left(1+C_{1}^{p}\right)\left(1+C_{2}^{p} \times \frac{t}{\operatorname{Re}}\right)\right] .
$$

The observed evolution of the pressure amplitude can be approximated by this form with sufficient accuracy, except for near the initial state. This approximation includes three coefficients: $C_{0}^{p}, C_{1}^{p}$ and $C_{2}^{p}$. The value of $C_{0}^{p}$ characterizes the increase in the pressure amplitude due to the divergence of the viscous terms; it is equal to unity when the divergence is zero. $C_{1}^{p}$ and $C_{2}^{p}$ characterize the decay rate and the rate of the temporal change in the decay rate of the pressure amplitude, respectively; their exact values are zero. As shown in Fig. 5(b), the value of these coefficients depends on the grid spacing, $\Delta x$. When $\Delta x$ is small, the deviation of the coefficients from the exact value is increased as the spatial resolution improves. Of the three coefficients, $C_{0}^{p}$ is the most sensitive to improvements in the grid spacing.

\subsection{Equilibrium property}

The divergence of the viscous terms affects the independence seen in the exact solution. The independence of the equilibrium relations is a characteristic of the exact solution which we use. The effect of the divergence of the viscous terms on the independence is examined by focusing on the equilibrium relation in the diffusion equation. When this independence holds, the spatial average of the square each side of the diffusion equation yields the following:

$$
\left\langle(\partial u / \partial t)^{2}\right\rangle^{1 / 2}=\left\langle\left((1 / \operatorname{Re})\left(\partial^{2} u / \partial x_{k}^{2}\right)\right)^{2}\right\rangle^{1 / 2}=(2 / \operatorname{Re}) \times \exp (-2 t / \operatorname{Re}) .
$$

Figure 6(a) shows the spatial rms average of the left- and right-hand sides of the diffusion equation, $\left\langle(\partial u / \partial t)^{2}\right\rangle^{1 / 2}$ and $\left\langle\left((1 / \operatorname{Re})\left(\partial^{2} u / \partial x_{k}^{2}\right)\right)^{2}\right\rangle^{1 / 2}$, respectively. Here, $N=128$ and $\epsilon_{p}=1$, and the divergence of the viscous terms is the largest of the computational conditions. We can see that $\left\langle(\partial u / \partial t)^{2}\right\rangle^{1 / 2}$ does not agree with $\left\langle\left((1 / \operatorname{Re})\left(\partial^{2} u / \partial x_{k}^{2}\right)\right)^{2}\right\rangle^{1 / 2}$ in the decay of the flow field. In addition, neither $\left\langle(\partial u / \partial t)^{2}\right\rangle^{1 / 2}$ nor $\left\langle\left((1 / \operatorname{Re})\left(\partial^{2} u / \partial x_{k}^{2}\right)\right)^{2}\right\rangle^{1 / 2}$ agrees with the exact solution. These disagreements show that, due to the divergence of the viscous terms, independence does not hold. As shown in the figure, the value of $\left\langle(\partial u / \partial t)^{2}\right\rangle^{1 / 2}$ is larger than that of $\left\langle\left((1 / \operatorname{Re})\left(\partial^{2} u / \partial x_{k}^{2}\right)\right)^{2}\right\rangle^{1 / 2}$. This suggests that the loss of independence may be caused by the deviation of $\left\langle(\partial u / \partial t)^{2}\right\rangle^{1 / 2}$. 

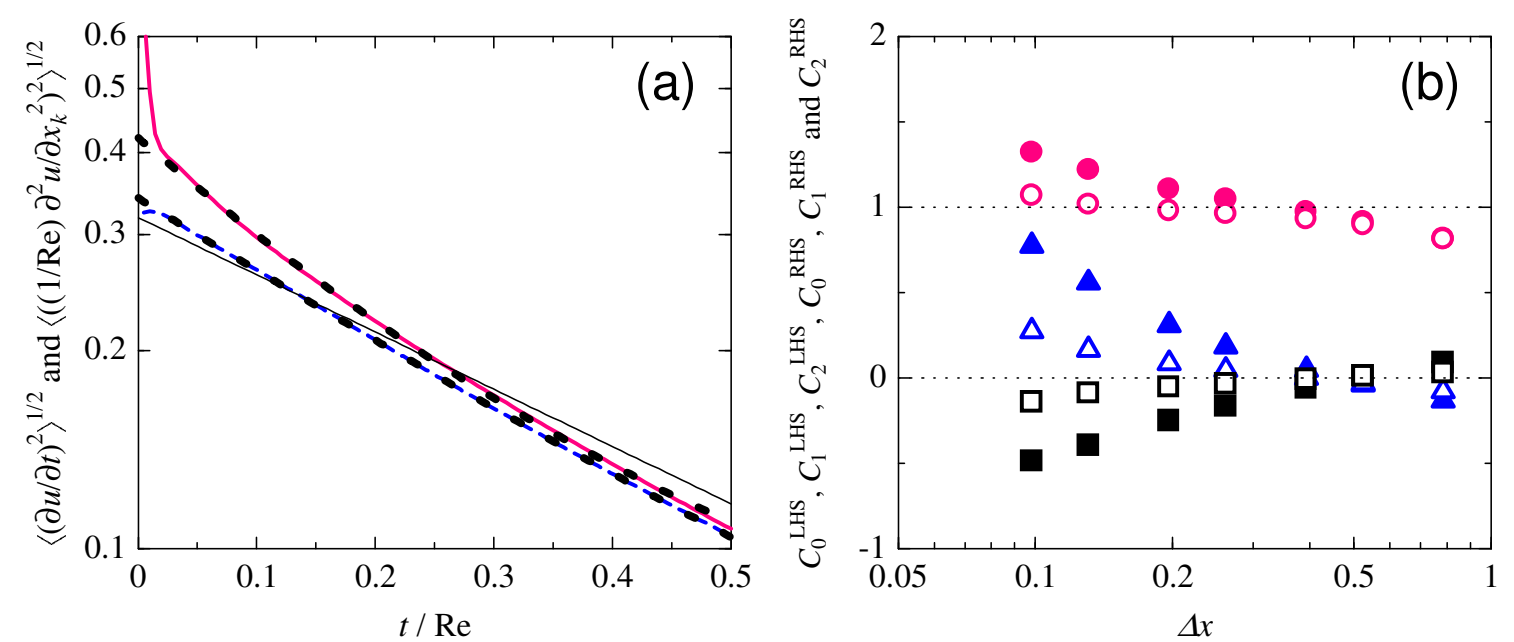

Fig. 6 Effects of the divergence of the viscous terms on the independence of the exact solution. (a) Temporal evolution of $\left\langle(\partial u / \partial t)^{2}\right\rangle^{1 / 2}$ and $\left\langle\left((1 / \operatorname{Re}) \partial^{2} u / \partial x_{k}^{2}\right)^{2}\right\rangle^{1 / 2}$. The black solid lines show the exact solution, and the red solid and blue dashed lines show the results of $\left\langle(\partial u / \partial t)^{2}\right\rangle^{1 / 2}$ and $\left\langle\left((1 / \operatorname{Re}) \partial^{2} u / \partial x_{k}^{2}\right)^{2}\right\rangle^{1 / 2}$, respectively. The dotted lines show the approximation given by Eq. (6) for the temporal evolution. The temporal evolutions do not agree with the exact solution, due to the divergence of the viscous terms. In addition, the temporal evolution of $\left\langle(\partial u / \partial t)^{2}\right\rangle^{1 / 2}$ does not agree with that of $\left\langle\left((1 / \operatorname{Re}) \partial^{2} u / \partial x_{k}\right)^{2}\right\rangle^{1 / 2}$. The disagreement shows that there is a nonequilibrium state between these values, and the dependency between the equilibrium relations arises due to the divergence of the viscous terms. The approximations given by Eq. (6) describe the temporal variations of these quantities. These approximations specify the effect of the divergence on these two quantities. Each approximation has three coefficients, the value of which is calculated by fitting it to the variations. (b) Values of the three coefficients. The red filled circles, blue filled triangles and black filled squares show the results for $C_{0}^{\mathrm{LHS}}, C_{1}^{\mathrm{LHS}}$ and $C_{2}^{\mathrm{LHS}}$, respectively. The open red circles, open blue triangles and open black squares show the results for $C_{0}^{\mathrm{RHS}}, C_{1}^{\mathrm{RHS}}$ and $C_{2}^{\mathrm{RHS}}$, respectively. As shown in the figure, the value of these coefficients depends on the grid spacing. The deviation of the two quantities is increased as the spatial resolution improves. $\left\langle(\partial u / \partial t)^{2}\right\rangle^{1 / 2}$ is more sensitive to the divergence of the viscous terms.

We now introduce an approximation for use when discussing the results of the loss of independence on the kinetic energy. We also introduce approximations for the terms $\left\langle(\partial u / \partial t)^{2}\right\rangle^{1 / 2}$ and $\left\langle\left((1 / \operatorname{Re})\left(\partial^{2} u / \partial x_{k}^{2}\right)\right)^{2}\right\rangle^{1 / 2}$, as follows:

$$
\begin{array}{ll}
\left\langle(\partial u / \partial t)^{2}\right\rangle^{1 / 2} & =C_{0}^{\mathrm{LHS}} \times(2 / \mathrm{Re}) \exp \left(-(2 t / \mathrm{Re})\left(1+C_{1}^{\mathrm{LHS}}\right)\left(1+C_{2}^{\mathrm{LHS}} \times(t / \mathrm{Re})\right)\right) \text { and } \\
\left\langle\left((1 / \mathrm{Re})\left(\partial^{2} u / \partial x_{k}^{2}\right)\right)^{2}\right\rangle^{1 / 2}= & C_{0}^{\mathrm{RHS}} \times(2 / \mathrm{Re}) \exp \left(-(2 t / \mathrm{Re})\left(1+C_{1}^{\mathrm{RHS}}\right)\left(1+C_{2}^{\mathrm{RHS}} \times(t / \mathrm{Re})\right)\right) .
\end{array}
$$

Here, $C_{0}^{\mathrm{LHS}}, C_{1}^{\mathrm{LHS}}$ and $C_{2}^{\mathrm{LHS}}$ are the effects of $\left\langle(\partial u / \partial t)^{2}\right\rangle^{1 / 2}$ on the intercept, decay rate and temporal rate of change of the decay rate, respectively. Similarly, $C_{0}^{\mathrm{RHS}}, C_{1}^{\mathrm{RHS}}$ and $C_{2}^{\mathrm{RHS}}$ are the respective effects of $\left\langle\left((1 / \mathrm{Re})\left(\partial^{2} u / \partial x_{k}^{2}\right)\right)^{2}\right\rangle^{1 / 2}$ on the same three quantities. The approximations fitted to the numerical results of these two terms are shown in the figure. We can see that, in the region of interest, the fitted formulae approximate the numerical results with sufficient accuracy.

The unsteady term, which formulates the rate of temporal change of the velocity, is more sensitive to the divergence of the viscous terms. By fitting the approximations to the numerical results, we calculated the value of each of the six coefficients. Figure 6(b) shows how the six coefficients depend on the spatial resolution. When the grid spacing is large, the deviation from the exact solution of the unsteady and viscous terms is comparable. This suggests that independence holds, although when the grid spacing is large, the numerically obtained flow field deviates from that of the exact solution. When the grid spacing is small, the divergence of the viscous terms is significant, and the deviation of the unsteady terms does not agree with that of the viscous terms. This is seen in all three quantities. The deviation of the intercept and the decay rate due to the divergence of the viscous terms is positive, and the deviation of the temporal rate of change of the decay rate is negative. The absolute magnitude of the deviation in the unsteady term is larger than that in the viscous terms. Therefore, we see that the unsteady term is more sensitive to the divergence of the viscous terms. Of the three terms, the absolute magnitude of the deviation of the decay rate is the largest, and thus we conclude that it is the most sensitive to the divergence of the viscous terms. 

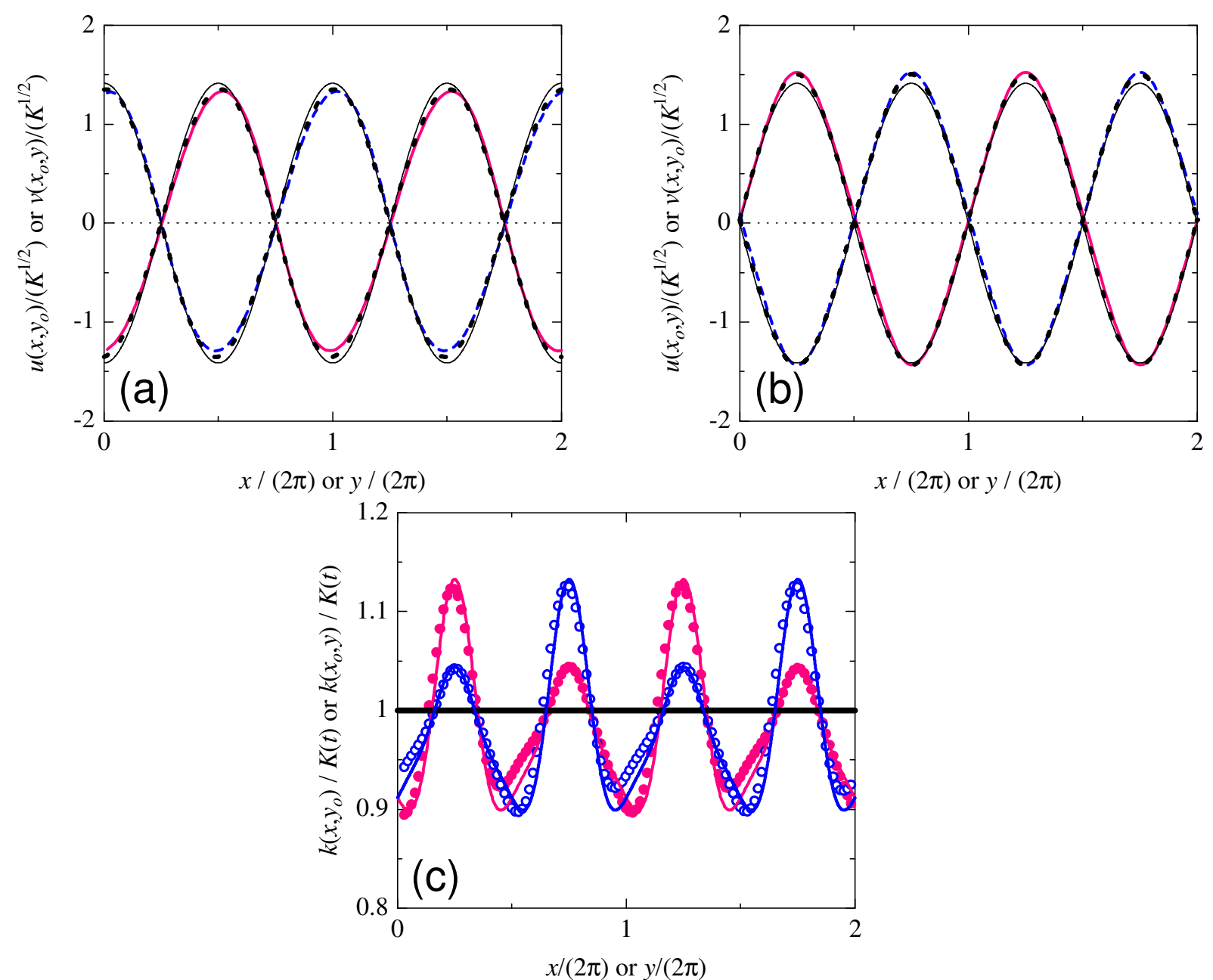

Fig. 7 Spatial variations of the velocities and kinetic energy, which are affected by the divergence of the viscous terms and harmonic waves (which are caused by the divergence), where $N=128$ and $\epsilon_{p}=1$. (a) Longitudinal variation of the velocities, where $x_{o}=y_{o}=\pi / 4$. The red solid and blue dashed lines show the results for $u\left(x, y_{o}\right) / K^{1 / 2}$ and $v\left(x_{o}, y\right) / K^{1 / 2}$, respectively. (b) Lateral variation. The red solid and blue dashed lines show the results of $u\left(x_{o}, y\right) / K^{1 / 2}$ and $v\left(x, y_{o}\right) / K^{1 / 2}$, respectively. In (a) and (b), the black solid lines show the variation in the velocity for the exact solution. The dotted lines show the approximation given in Eq. (8); this approximation can explain the variation in velocity due to the divergence. (c) Spatial variation of the kinetic energy, as affected by the divergence. The red filled and open blue symbols show $k\left(x, y_{o}\right) / K(t)$ and $k\left(x_{o}, y\right) / K(t)$, respectively. The variations in the kinetic energy, including these harmonic waves, are described by Eq. (8).

\subsection{Harmonic wave}

The divergence of the viscous terms causes additional harmonic waves in the flow field. We compared the flow field that is affected by the divergence of the viscous terms to that without the effect of the divergence. Figures 7(a) and (b) show the spatial variation in the flow field for both the longitudinal and lateral directions. Here, $x_{o}=y_{o}=\pi / 4, N=128$ and $\epsilon_{p}=1$. As shown in the figure, the flow field of the numerical results does not agree with that of the exact solution. This disagreement is found in the longitudinal and lateral variations of the velocity field. In the longitudinal variation, the deviation of the numerical results is significant around positions at which the value of the variation is an extremum. In the lateral variation, the deviation is significant when the value is a positive extremum, and the lateral variations are skewed. We assume that these deviations indicate an additional flow field caused by the divergence of the viscous terms. To examine the additional flow field, we focus on the variation in the kinetic energy. The kinetic energy of the exact solution at $x_{o}=\pi / 4$ or $y_{o}=\pi / 4$ satisfies

$$
k\left(x, y_{o}\right) / K(t)=1 \text { or } k\left(x_{o}, y\right) / K(t)=1
$$

That is, the kinetic energy normalized by $K(t), k(x, y) / K(t)$, is unity along the lines in the flow field of the exact solution. Figure 7(c) shows the kinetic energy of the numerical results at $x_{o}=\pi / 4$ or $y_{o}=\pi / 4$. The kinetic energy of the numerical results does not agree with that of the exact solution, and it indicates the existence of harmonic waves. 

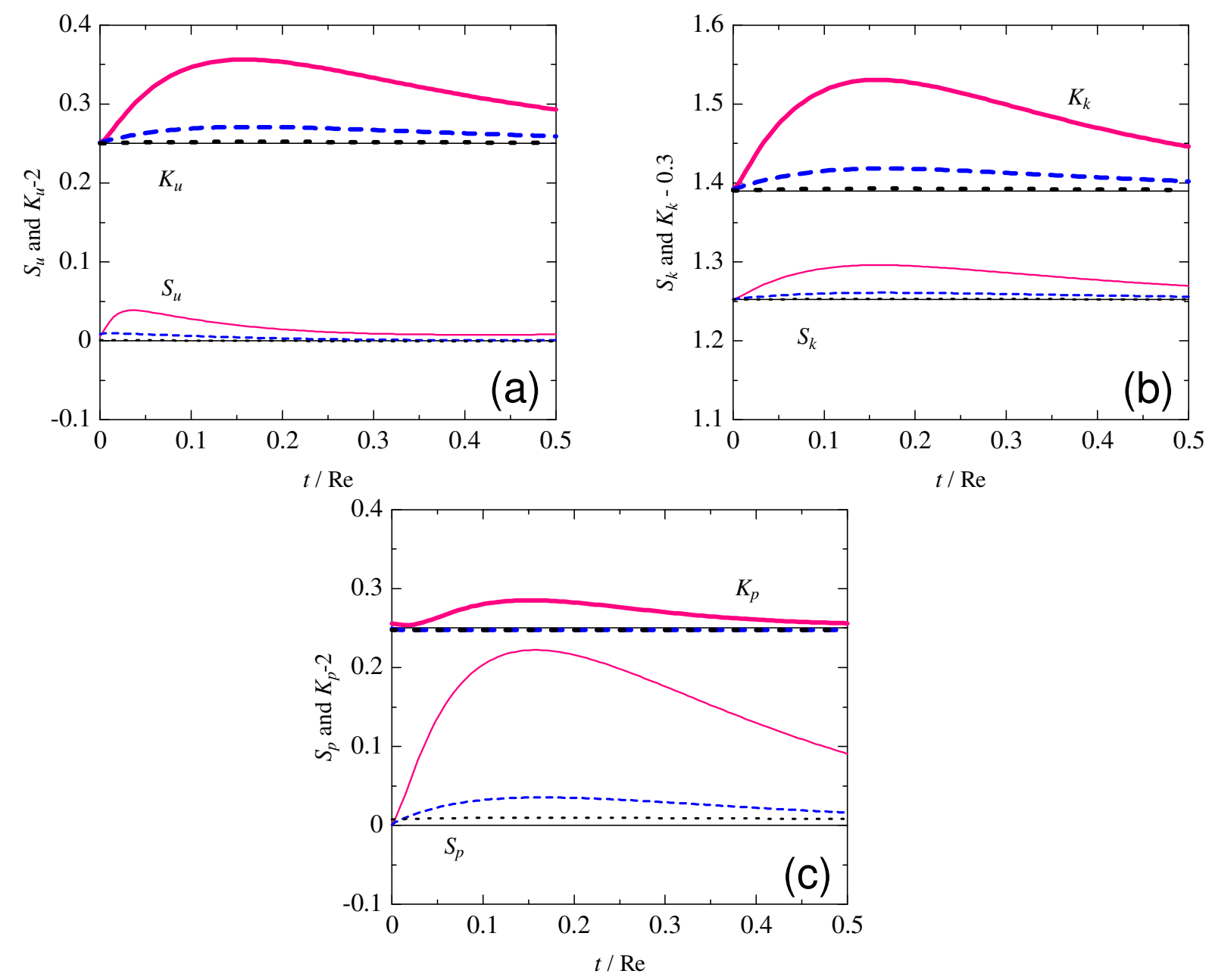

Fig. 8 Temporal evolution of the skewness and kurtosis of (a) velocity $u$, (b) kinetic energy $k$ and (c) pressure $p$. Here, $S_{u}, S_{k}$ and $S_{p}$, and $K_{u}, K_{k}$ and $K_{p}$ are the skewness and kurtosis factors of the velocity $(u)$, pressure $(p)$ and kinetic energy $(k)$, respectively. Thin and thick lines indicate the skewness and kurtosis factors, respectively. Red solid, blue dashed and black dotted lines indicate the results with $\epsilon_{p}=1,0.1$ and 0.01 , respectively. The thin black lines show the exact values of the factors shown in Eq. (10). When the value of $\epsilon_{p}$ is not small, the divergence of the viscous terms is not small, and thus the factors deviate from the corresponding exact value. This suggests that the divergence of the viscous terms affects the shape of the spatial variation of the physical quantities.

We now consider the wave number of the harmonic waves that are caused by the divergence of the viscous terms; to do so, we will use an approximation of the numerical flow field. The longitudinal and lateral variations of the velocity field normalized by the square root of $K(t)$ can be approximated as follows:

$$
\begin{aligned}
& \frac{u\left(x, y_{o}\right)}{K^{1 / 2}}=a_{1}^{f} \cos (x)+b_{2}^{f} \sin (2 x) \text { or } \frac{v\left(x_{o}, y\right)}{K^{1 / 2}}=-a_{1}^{f} \cos (y)-b_{2}^{f} \sin (2 y) \text { and } \\
& \frac{u\left(x_{o}, y\right)}{K^{1 / 2}}=a_{0}^{g}+a_{1}^{g} \sin (y)+b_{3}^{g} \sin (3 y) \text { or } \frac{v\left(x, y_{o}\right)}{K^{1 / 2}}=a_{0}^{g}-a_{1}^{g} \sin (x)-b_{3}^{g} \sin (3 x) .
\end{aligned}
$$

Here, $a_{1}^{f}=-1.35, b_{2}^{f}=0.04, a_{0}^{g}=0.03, a_{1}^{g}=1.45$ and $b_{3}^{g}=-0.025$. Figures 7 (a) and (b) validate the approximations of the velocity variations; that is, it can be seen that these approximations are sufficiently accurate. Using these approximations, an approximation of the kinetic energy is also derived. The derived approximation is seen to be validated in (c). We consider the form of the approximation in order to evaluate the wave number of the harmonic waves due to the divergence of the viscous terms. We will use two trigonometric functions to approximate the longitudinal variation, and we will assume that the second term is caused by the divergence of the viscous terms. Further, we assume that the wave number of the primary term is equal to that of the exact solution, and the wave number of the secondary term is greater than that of the exact solution. The approximation of the lateral variation consists of three parts: a mean term, a $\sin (3 y)$ term, and a $\cos (3 x)$ term. Our results show that the wave number is different for the longitudinal and lateral variations, and the wave number of the lateral variation is indeed greater than that of the longitudinal variation. 

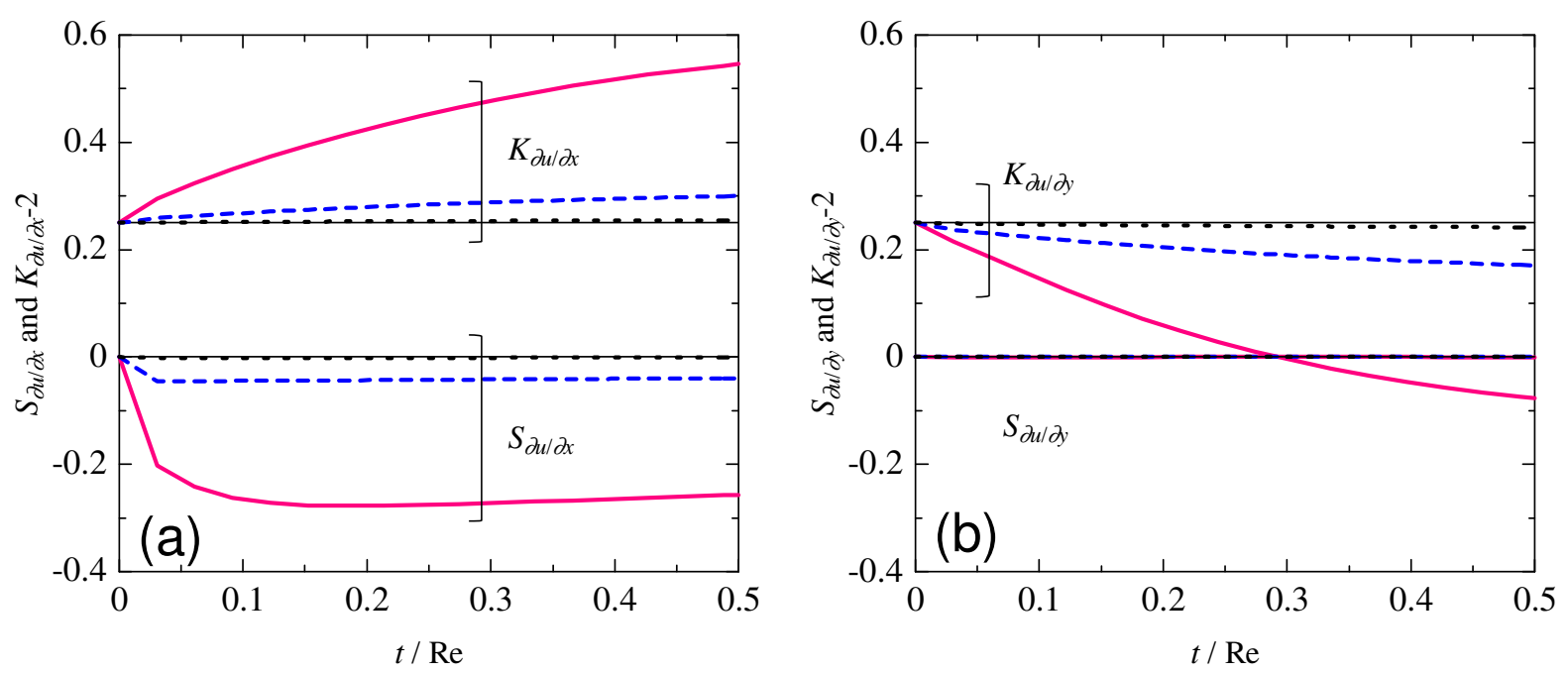

Fig. 9 Temporal evolution of the skewness and kurtosis of the (a) longitudinal $(\partial u / \partial x)$ and (b) lateral $(\partial u / \partial y)$ velocity gradients. Here, $S_{\partial u / \partial x}$ and $S_{\partial u / \partial y}$ are the skewness factors of the velocity gradients, which are defined in Eq. (11), and $K_{\partial u / \partial x}$ and $K_{\partial u / \partial y}$ are the kurtosis factors of the velocity gradients, which are defined in Eq. (12). Red solid, blue dashed and black dotted lines show the results for $\epsilon_{p}=1,0.1$ and 0.01 , respectively. The thin black lines show the exact values of the factors shown in Eqs. (11) and (12). The skewness and kurtosis factors are sensitive to the divergence of the viscous terms, except for the skewness of the lateral gradient of the velocity field.

\subsection{Distribution profile}

We now examine the shape of the spatial distribution of the physical quantities and use exact forms to characterize the distribution. The divergence of the viscous terms causes an additional flow field. We also examine the spatial distribution of the physical quantities affected by the divergence of the viscous terms. Third-order and fourth-order spatial averages of the velocity $u$, kinetic energy $k$ and pressure $p$ fields are formed as follows:

$$
\begin{aligned}
& \left\langle u^{3}\right\rangle=0,\left\langle k^{3}\right\rangle=(7 / 4) \exp (-12 t / \operatorname{Re}),\left\langle p^{3}\right\rangle=0, \\
& \left\langle u^{4}\right\rangle=(9 / 4) \exp (-16 t / \mathrm{Re}),\left\langle k^{4}\right\rangle=(169 / 100) \exp (-16 t / \mathrm{Re}) \text { and }\left\langle p^{4}\right\rangle=(9 / 64) \exp (-16 t / \mathrm{Re}) .
\end{aligned}
$$

Using these formulations, we define the following quantities:

$$
\begin{aligned}
& S_{u}=\frac{\left\langle u^{3}\right\rangle}{\left\langle u^{2}\right\rangle^{3 / 2}}=0, S_{k}=\frac{\left\langle k^{3}\right\rangle}{\left\langle k^{2}\right\rangle^{3 / 2}}=\frac{14}{5 \sqrt{5}}, S_{p}=\frac{\left\langle p^{3}\right\rangle}{\left\langle p^{2}\right\rangle^{3 / 2}}=0, \\
& K_{u}=\frac{\left\langle u^{4}\right\rangle}{\left\langle u^{2}\right\rangle^{2}}=\frac{9}{4}, K_{k}=\frac{\left\langle k^{3}\right\rangle}{\left\langle k^{2}\right\rangle^{3 / 2}}=\frac{169}{100} \text { and } K_{p}=\frac{\left\langle p^{4}\right\rangle}{\left\langle p^{2}\right\rangle^{2}}=\frac{9}{4} .
\end{aligned}
$$

We assume that these quantities characterize the shape of the spatial distribution. The value of $S_{u}, S_{k}$ and $S_{p}$ characterize the skew of the spatial distribution, and the spread of the spatial distribution is characterized by the values of $K_{u}, K_{k}$ and $K_{p}$, which correspond to the kurtosis. Below, we examine the effect of the divergence in the viscous terms on the skewness and kurtosis.

The divergence of the viscous terms increases the magnitudes of the skewness and kurtosis of the spatial distribution of the physical quantities. Figure 8 shows the effect of the divergence on the skewness and kurtosis of the velocity $u$, kinetic energy $k$ and pressure $p$. The skewness due to the divergence of the viscous terms decreases as time increases, except around the initial state; this occurs because the divergence of the viscous terms is reduced as time increases. The skewness of the pressure is the most sensitive to the divergence of the viscous terms. The deviation of the skewness due to the divergence can be reduced by increasing convergence. Similarly, the kurtosis is also affected by the divergence of the viscous terms, and of the three quantities, the kurtosis of the kinetic energy is the most sensitive to the divergence. The deviation of the kurtosis can also be reduced by increasing convergence.

The spatial average of the gradients of the velocity fields is also affected by the divergence of the viscous terms. The skewness and kurtosis of the gradients of the velocity fields have frequently been examined in previous studies of turbulent flow. We investigated the effect of the divergence of the viscous terms on these factors. In the exact solution, the skewness and kurtosis of the velocity gradients are derived as follows:

$$
S_{\partial u / \partial x}=S_{\partial u / \partial y}=S_{\partial v / \partial x}=S_{\partial v / \partial y}=0, \text { where }
$$




$$
\begin{aligned}
S_{\partial u / \partial x} & =\frac{\left\langle(\partial u / \partial x)^{3}\right\rangle}{\left\langle(\partial u / \partial x)^{2}\right\rangle^{3 / 2}}, S_{\partial u / \partial y}=\frac{\left\langle(\partial u / \partial y)^{3}\right\rangle}{\left\langle(\partial u / \partial y)^{2}\right\rangle^{3 / 2}}, S_{\partial v / \partial x}=\frac{\left\langle(\partial v / \partial x)^{3}\right\rangle}{\left\langle(\partial v / \partial x)^{2}\right\rangle^{3 / 2}}, S_{\partial v / \partial y}=\frac{\left\langle(\partial v / \partial y)^{3}\right\rangle}{\left\langle(\partial v / \partial y)^{2}\right\rangle^{3 / 2}} . \\
K_{\partial u / \partial x} & =K_{\partial u / \partial y}=K_{\partial v / \partial x}=K_{\partial v / \partial y}=9 / 4, \text { where } \\
K_{\partial u / \partial x} & =\frac{\left\langle(\partial u / \partial x)^{4}\right\rangle}{\left\langle(\partial u / \partial x)^{2}\right\rangle^{2}}, K_{\partial u / \partial y}=\frac{\left\langle(\partial u / \partial y)^{4}\right\rangle}{\left\langle(\partial u / \partial y)^{2}\right\rangle^{2}}, K_{\partial v / \partial x}=\frac{\left\langle(\partial v / \partial x)^{4}\right\rangle}{\left\langle(\partial v / \partial x)^{2}\right\rangle^{2}}, K_{\partial v / \partial y}=\frac{\left\langle(\partial v / \partial y)^{4}\right\rangle}{\left\langle(\partial v / \partial y)^{2}\right\rangle^{2}} .
\end{aligned}
$$

Here, the values of the skewness and kurtosis are the same in both the longitudinal and lateral directions. Figure 9 shows the temporal profiles of the skewness and kurtosis of the velocity gradients. The divergence of the viscous terms reduces the skewness of the longitudinal gradient of the velocity field, although the divergence has little effect on the skewness of the lateral gradient. The reduction in the skewness of the longitudinal gradient is on the order of 0.1 ; we note that the skewness of the velocity gradient in a turbulent flow is commonly about -0.5 . Thus, the reduction in the skewness is comparable to that found in turbulent flows. The kurtosis of the velocity gradient is also affected by the divergence of the viscous terms, which increase the kurtosis of the longitudinal gradient and decrease that of the lateral gradient.

\section{Conclusion}

The present study uses the exact solution to examine the effect of the divergence of the viscous terms on the incompressible flow. Although the divergence of the viscous terms is exactly zero in the exact solution of an incompressible flow, when an iterative method is applied to solving the Poisson equation, the divergence of the viscous terms can be nonzero. In addition, the divergence of the viscous terms can be larger than that of the velocity when the spatial resolution is high and the Reynolds number is small. Exact solutions have often been used as benchmarks for numerical simulations. We used the exact solution, which is well known and has been widely used, to investigate the effects of the divergence of the viscous terms. The reasons for using the exact solution is that the flow field is perfectly known and is described by a mathematical formula. We focus on the fact that the two equilibrium relations in the exact solution are independent. The divergence of the viscous terms affects both the flow field of the exact solution and the independence. The numerical conditions in this study were carefully set so as to clarify the effects of the divergence of the viscous terms. For instance, the value of the CFL condition was fixed for the spatial resolution and was smaller than unity. This allowed us to clarify the effects caused by the divergence of the viscous terms.

The divergence of the viscous terms significantly affects the flow field of the exact solution. The decay rate of the kinetic energy is increased by the divergence of the viscous terms. The independence of the equilibrium relations and pressure are also affected by the divergence in the flow field. These uncertainties are quantitatively examined by approximating the exact solution. The divergence also results in harmonic waves in the flow field. The wave number of the longitudinal velocity of the harmonic waves is different from that of the lateral velocity. The skewness and kurtosis factors of the velocity, kinetic energy, and pressure in the flow field are also affected by the divergence of the viscous terms; their divergence increases. Although the effects of the divergence on the exact solution are rather large, as shown in the figure, the effects of the divergence can be reduced by improving the convergence of the Poisson equation. The accuracy of simulations of incompressible flow can be improved by reducing the divergence of the viscous terms as well as that of the velocity.

Acknowledgments The authors would like to thank Mr. Hayato Ikeda (Yamaguchi University) for his assistance in conducting the simulations. The present study was supported in part by the Japanese Ministry of Education, Culture, Sports, Science and Technology through Grants-in-Aid (Nos. 15K13871 and 15K17970).

\section{References}

Addad, Y., Zaidi, I. and Laurence, D., Quasi-DNS of natural convection flow in a cylindrical annuli with an optimal polyhedral mesh refinement, Computers \& Fluids, Vol.118 (2015), pp.44-52.

Brachet, M. E., Meiron, D. I., Orszag, S. A., Nickel, B. G., Morf, R. H. and Frisch, U., Small-scale structure of the Taylor-Green vortex, Journal of Fluid Mechanics, Vol.130 (1983), pp.411-452.

Brachet, M. E., Direct simulation of three-dimensional turbulence in the Taylor-Green vortex, Fluid Dynamics Research, Vol.8, No.1-4 (1991), pp.1-8.

Ethier, C. R. and Steinman, D. A., Exact fully 3D Navier-Stokes solutions for benchmarking, International Journal for Numerical Methods in Fluids, Vol.19, No.5 (1994), pp.369-375. 
Gotoh, T., Hatanaka, S. and Miura, H., Spectral compact difference hybrid computation of passive scalar in isotropic turbulence, Journal of Computational Physics, Vol.231, No.21 (2012), pp.7398-7414.

Hui, W. H., Exact solutions of the unsteady two-dimensional Navier-Stokes equations, Journal of Applied Mathematics and Physics, Vol.38, No.5 (1987), pp.689-702.

Kato, C., Yamade, Y., Wang, H., Guo, Y., Miyazawa, M., Takaishi, T., Yoshimura, S. and Takano, Y., Numerical prediction of sound generated from flows with a low Mach number, Computers \& Fluids, Vol.36, No.1 (2000), pp.53-68.

Kim, J. and Moin, P., Application of a fractional-step method to incompressible Navier-Stokes equations, Journal of Computational Physics, Vol.59, No.2 (1985), pp.308-323.

Komen, E., Shams, A., Camilo, L. and Koren, B., Quasi-DNS capabilities of OpenFOAM for different mesh types, Computers \& Fluids, Vol.96 (2014), pp.87-104.

Lamballais, E., Fortune, V. and Laizet, S., Straightforward high-order numerical dissipation via the viscous term for direct and large eddy simulation, Journal of Computational Physics, Vol.230, No.9 (2011), pp.3270-3275.

Miyauchi, T., Hirata T. and Tanahashi M., Direct numerical simulation of two-dimensional homogeneous isotropic turbulence by higher-order finite difference scheme (Comparison with the spectral method), Transactions of the JSME ser.B, Vol.60, No.574 (1994), pp.1999-2006. (in Japanese)

Morinishi, Y., Lund, T. S., Vasilyev, O. V. and Moin, P., Fully conservative higher order finite difference schemes for incompressible flow, Journal of Computational Physics, Vol.143, No.1 (1998), pp.90-124.

Morinishi, Y., Ichikawa, A., Okumura, T. and Nabayashi, K., Effects of the order of accuracy of the finite-difference method and grid resolution on LES of wall turbulence, Transactions of the JSME ser.B, Vol.66, No.647 (2000), pp.136-143. (in Japanese)

Rogler, H. L. and Reshotko, E., Spatially decaying array of vortices, Physics of Fluids, Vol.19, No.12 (1976), pp.18431850.

San, O. and Staples, A. E., A coarse-grid projection method for accelerating incompressible flow computations, Journal of Computational Physics, Vol.233 (2013), pp.480-508.

Suzuki, H., Nagata, K., Sakai, Y. and Hayase, T., Direct numerical simulation of turbulent mixing in regular and fractal grid turbulence, Physica Scripta, Vol.2010, No.T142 (2010), 014065.

Suzuki, H., Nagata, K., Sakai, Y., Hayase, T., Hasegawa, Y. and Ushijima, T., An attempt to improve accuracy of higherorder statistics and spectra in direct numerical simulation of incompressible wall turbulence by using the compact schemes for viscous terms, International Journal for Numerical Methods in Fluids, Vol.73, No.6 (2013a), pp.509-522.

Suzuki, H., Nagata, K., Sakai, Y., Hayase, T., Hasegawa, Y. and Ushijima, T., Direct numerical simulation of fractalgenerated turbulence, Fluid Dynamics Research, Vol.45, No.6 (2013b), 061409.

Tamura, A., Kikuchi, K. and Takahashi, T., Residual cutting method for elliptic boundary value problems, Journal of Computational Physics, Vol.137, No.2 (1997), pp.247-264.

Wang, C. Y., Exact solutions of the Navier-Stokes equations-the generalized Beltrami flows, Acta Mechanica, Vol.81, No.1 (1990), pp.69-74.

Wang, C. Y., Exact solutions of the steady-state Navier-Stokes equations, Annual Review of Fluid Mechanics, Vol.23, No.1 (1991), pp.159-177.

Zhou, Y., Nagata, K., Sakai, Y., Suzuki, H., Ito, Y., Terashima, O. and Hayase, T., Development of turbulence behind the single square grid, Physics of Fluids, Vol.26, No.4 (2014a), 045102.

Zhou, Y., Nagata, K., Sakai, Y., Suzuki, H., Ito, Y., Terashima, O. and Hayase, T., Relevance of turbulence behind the single square grid to turbulence generated by regular-and multiscale-grids, Physics of Fluids, Vol.26, No.7 (2014b), 075105 . 